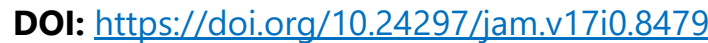

\title{
Natural Mathematics, the Fibonacci Numbers and Aesthetics in Art
}

\author{
Seamus A. Power ${ }^{\mathrm{a}}$ and Anthony G. Shannon ${ }^{\mathrm{b}}$ \\ ${ }^{a} 63$ Effingham Street, Tarragindi, QLD 4121, Australia \\ bFellow, Warrane College, the University of New South Wales, PO Box 123, Kensington, NSW 1465, Australia \\ awtel.power@gmail.com, t.shannon@warrane.unsw.edu.au
}

\begin{abstract}
The Mathematics of beauty and beauty in mathematics are important ingredients in learning in the liberal arts. The Fibonacci numbers play an important and useful role in this. This paper seeks to present and illustrate a grounding of visual aesthetics in natural mathematical principles, centered upon the Fibonacci numbers. The specific natural mathematical principles investigated are the Fibonacci numbers, the Fibonacci Spiral, and the Cosmic Bud.
\end{abstract}

Indexing terms/Keyword: Fibonacci Numbers, Recurrence Relation, Curvature, Fibonacci Spiral Golden Rectangle.

Academic Discipline and Sub-discipline: Mathematics: Number Theory, Art

Subject classification: 00A66 [Mathematics and visual arts, visualization], 11B39 [ Fibonacci and Lucas numbers and generalizations]

\section{Type (method/approach)}

This paper outlines some of the connections of the sequence of Fibonacci numbers with visual art. It does this partly by reference to key references in the literature, but mainly by taking examples which demonstrate possible connections are well-known visualizations of Fibonacci spirals. Some of these illustrations are regarded as beautiful, but one of them is unexpectedly so and not by design.

\section{Introduction}

Beauty, Truth, and Goodness are the legs of a thorough educational tripod [1,2]. In particular, beauty in mathematics [3] and the mathematics of beauty [4] are important ingredients of sound teaching and cultured learning [5]. There is a broader societal problem, identified by the late great Russian-born mathematical educator, Alexander Bogomolny (1948-2018) that even among people with post-high school formal education it has "become an acceptable norm to confess a dislike and misunderstanding of Mathematics as a whole" and that lack of awareness of the beauty of mathematics and the mathematics of beauty are contributing factors: "judging Mathematics by its pragmatic value is like judging a symphony by the weight of its score" [6]!Moreover, it has been noted by authoritative figures in the art world [7], and the sentiment is more generally held, that art pieces from contemporary movements do not seem to invoke a naturally discernible aestheticism. This seems to be a view restricted specifically to more recent art movements. What has changed, it will be implied, is that pieces of art from more recent movements tend to lack a certain form - a form referencing natural mathematical principles, centered upon the Fibonacci numbers. This form, of course, has been more faithfully referred to in art pieces from the pre-modern movements.

Some items considered will include an exploration into art pieces from pre-modern movements famous for their widely renowned aestheticism. The exploration will uncover their Line, an Element of Art that closely follows, in various ways, literal and implied visual representations of the Fibonacci spiral. In addition to this Element of Art, 
the existence of Line of a different nature - Implied Line, will be posited, to allow for a broader scope within which a piece of art may be considered adhering to a Fibonacci spiral. To further illustrate the chief assertion of this piece, somewhat of an aesthetic oddity will be presented: a photo, taken with no intentional motivation of being aesthetically pleasing, is nevertheless so.

\section{The Fibonacci numbers}

\subsection{The Fibonacci sequence}

The Fibonacci sequence is an ordered set of integers, $\left\{F_{n}\right\}, n>0$, in which each integer is the sum of the previous two terms as represented by the second-order homogeneous linear recurrence relation

$F_{n}=F_{n-1}+F_{n-2,} n>2$,

with initial conditions $F_{1}=F_{2}=1$, so that the first nine terms are given by

$\left\{F_{n}\right\} \equiv\{1,1,2,3,5,8,13,21,34, \ldots\}$.

\subsection{The Fibonacci spiral}

The 'Fibonacci Spiral' (Figure 1) is found by the arrangement of rectangles whose side lengths are the Fibonacci numbers. The spiral is then drawn from the contiguous arrangements of the rectangles. The Fibonacci Spiral is especially important because when arranged in a particular way, a number of Fibonacci Spirals form a Cosmic Bud (Figure 2).

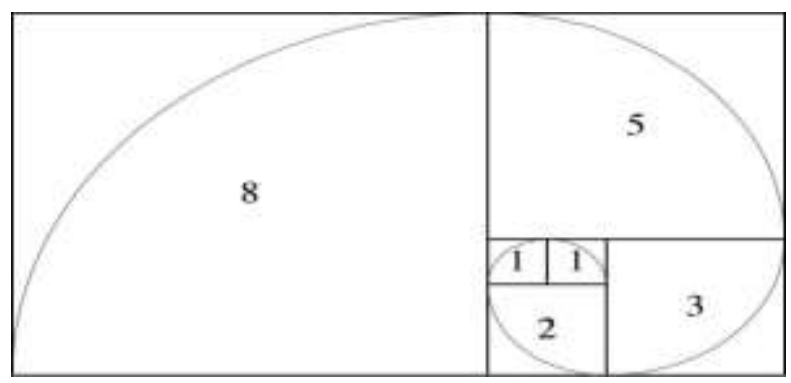

Figure 1: The Fibonacci Spiral

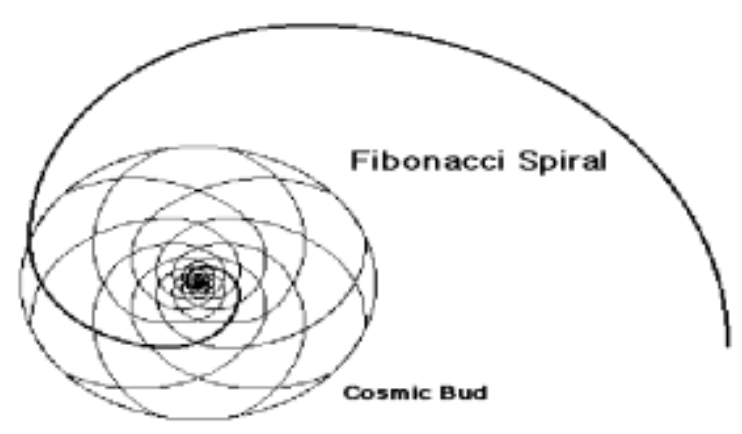

Figure 2: The Cosmic Bud, Composed from Multiple Fibonacci Spirals

The Cosmic Bud 'maps out' the seed configuration of a number of flowers, the arrangement of leaves in a number of succulents, the positioning of pines in a pine cone, and most notably, the inner structures in a number 
of seashells. It becomes apparent then, that wherever there exist patterns in the natural world with some level of complex beauty, one is likely to find the Fibonacci numbers and the Fibonacci spiral lurking somewhere underneath. The Fibonacci Spiral and Cosmic Bud, it is asserted, are two of the most fundamental aesthetic components of nature. It is the presence of these natural mathematical concepts which contribute to the unique laying of the structural foundation for aesthetically pleasing items of visual inspection.

Indeed, when the Fibonacci spiral is superimposed onto a select group of notable and genuinely aesthetically appealing artworks, one begins to see the causation between the conformity of an art piece to the Fibonacci spiral, and the work's overall objective aesthetic appeal.

3

\section{Line as an artistic element}

As one of the seven Elements of Art, Line pertains to certain markings that indicate a particular course traversed between two points [8]. It is important to note that the markings may be bi-directional - both in the implied forward and reverse directions along the line.

\subsection{Implied line}

Consider then, the possibility of an implied Element of Line. These implied manifestations of Line can be brought to the attention of the viewer by means outside of literal markings. The artist may choose to employ a series of certain landmarks in the work through which an implied Line may establish itself. The implied Line may, therefore 'traverse' through, for example, the centers of mass, or distinguishing features of human figures, along the edges of multiple separate objects, or through important features on the canvas. Additionally, it could be eluded to by the multiple pointed fingers, or the line of sight of human figures on the canvas.

\subsection{Classical art and the Fibonacci spiral}

\subsubsection{The great wave off Kanagawa}

Hokusai's colored woodblock print, The Great Wave off Kanagawa (Figure 3), published at around the turn of the third decade in the eighteenth century is possessive of perhaps the most openly apparent adherence to the Fibonacci spiral among artwork from the pre-modern age. The work is widely known, highly regarded, and authoritatively thought of as the archetypal example of Japanese art of the era, [9]. In examination of the piece, one discovers the close adherence of Line in the work to the visual representation of a Fibonacci spiral. When a Fibonacci spiral is positioned with its 'head' at the underside of the crest of the wave and rotated, very neat parallels become apparent.

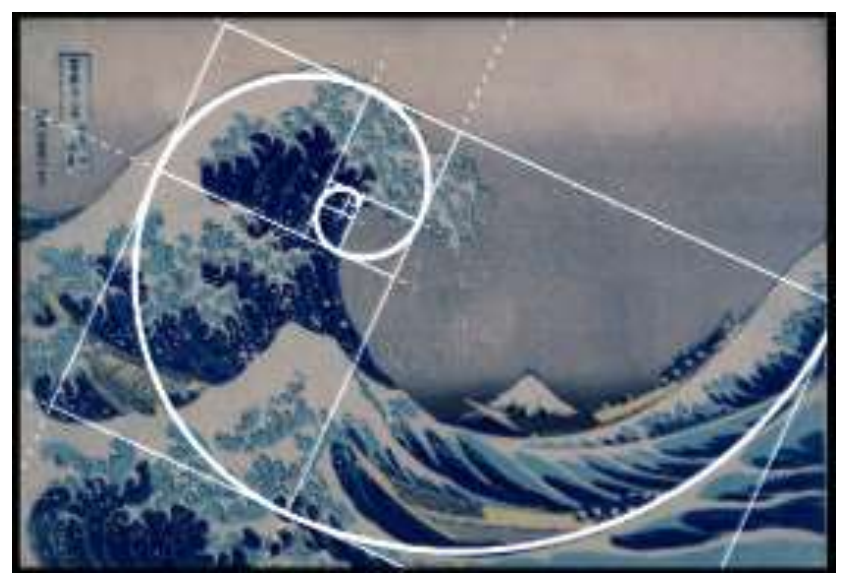

Figure 3: The Great Wave off Kanagawa

Image Credit: https://www.reddit.com/r/WallpaperRequests/comments/5ewxi1/hokusai_fibonacci/ 
The section of spiral denoted by the first seven Fibonacci 'boxes' neatly outlines the whole crest of the wave. The next section of the spiral, denoted by the eighth Fibonacci golden rectangle, traverses a line, whose angle, orientation, and curvature, when transposed to the right, follow in a near-perfect fashion, the ascending edge on the inside of the wave. Finally, there is depicted, the tail end of the preceding wave that disappears off to the right of the canvas. The top side of this wave, as it flattens out into the surface of the ocean, again faithfully follows the segment of Fibonacci spiral outlined by the ninth Fibonacci golden rectangle. This final segment of the implied Fibonacci Line is all the more interacted with by the work when the Japanese boat disappears behind the tail of this preceding wave. The interface that exists between the wave and the boat being partially obscured is neatly bisected by the implied Fibonacci spiral.

\subsubsection{Aldobrandini Madonna}

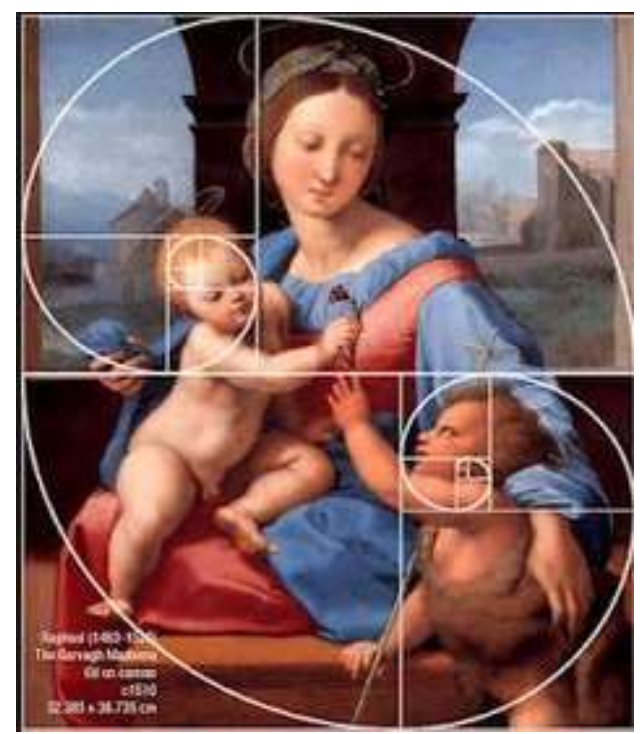

Figure 4: Aldobrandini Madonna

Image Credit: https://www.pinterest.com.au/pin/378654281140737993/

Painted by Renaissance artist Raphael, the Aldobrandini Madonna depicts Mary, the mother of Christ, holding both Christ in her right hand and John the Baptist in her left. Though there are less overt adherences to the Fibonacci spiral in this work when compared to The Great Wave off Kanagawa, there are faithful, subtle adherences to be discovered through the Implied Line of the work.

The work is seen to adhere to the lines scribed by two separate Fibonacci spirals. These spirals are found to be mirrored against each other both in the horizontal and vertical dimensions, and then one transposed below so that its upper edge borders with the lower edge of the higher spiral.

The head of the first spiral in the upper half of the work closely contours the top and right edges of the face of the infant Christ. It orientates the head to be pointed downwards to the face of the infant John the Baptist. This upper spiral then traverses through the center of the supporting hand of Mary and then acts as a concentric outer segment to her left neck through to the upper arm.

The lower spiral originates in the nape of the neck of the infant John the Baptist and courses through the two joints of the left upper arm. From the chin to the uppermost point of his head, the implied Fibonacci line traces the sweeping trajectory of the profile of his face, albeit the implied line sits just left of the profile. 


\subsection{Accidental aesthetic anomaly}

Take, for example, a photograph of a fight that had broken out in a Ukrainian parliamentary room in August 2014 (Figure 5). The photograph was taken by a political photographer and received an enormous cult internet following. Whilst not intended to be particularly aesthetically appealing but rather mere documentation of events, the photo has a genuine sense of aesthetic harmony intrinsic to it. Inspection shows that, by chance, the subjects of the photo were arranged and positioned in such a way that the image's Implied Line conformed to a Fibonacci spiral.
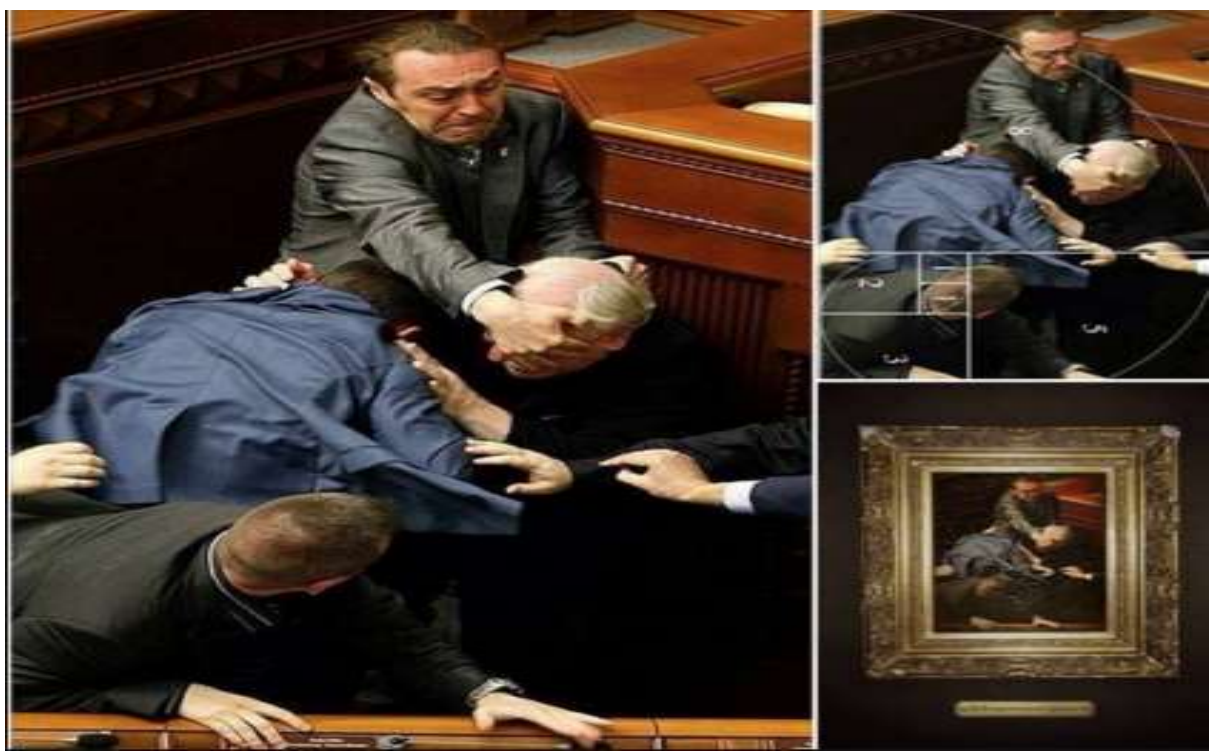

\section{Figure 5: Ukrainian parliamentary fight}

Image Credit: https://www.kyivpost.com/article/content/war-against-ukraine/the-guardian-ukrainian-parliament-the-photos-that-look-like-italian-paintings-359744.html

Though the original photo could not be located, a later edited version with a Fibonacci spiral already superimposed was. To further illustrate the point, the editor 'placed' the image in a traditional ornate frame. Guardian Journalist, Ben Beaumont-Thomas coined the term: Accidental Renaissance [10]. This term denoted certain candid photos that had a particular style similar to what could be found in Renaissance art. Upon closer inspection, these images just so happened to have elements of their implied line that were faithful to the Fibonacci spiral.

\section{Conclusion}

It is quite clear that the Fibonacci numbers, the Fibonacci Spiral and the Cosmic Bud are present in some naturally occurring manifestations of complex beauty. It is therefore also apparent that the Fibonacci numbers, the Fibonacci Spiral and Cosmic Bud can, in and of themselves, serve as a foundational structure for some manifestations of aesthetically appealing items of visual inspection. Additionally, it is conceivable that, given the existence of Line as an Artistic Element, the positing of the existence of an implied Line, whose existence is found through not readily apparent means, is relatively uncontroversial. Closer inspection of the works of Hokusai and Raphael identifies, through the medium of both Line and Implied Line, an adherence to Fibonacci Spiral. It is not considered likely that artists of notably aesthetically appealing works start from a skeletal Fibonacci spiral structure and develop their work thenceforth. It is, however, reasonable to consider, that, after the fact, pieces of work whose Line or implied Line are found to adhere to the Fibonacci Spiral, or multiple spirals, are likely to be considered aesthetically appealing. 


\section{Data Availability}

The data can be accessed through the bibliographic and url references.

\section{Conflicts of Interest}

The authors declare that that there are no conflicts of interest regarding the publication of this article.

\section{Funding Statement}

The research was funded by the authors themselves.

\section{References}

1. Gardner, Howard, 2011. Truth, beauty, and goodness reframed: Educating for the virtues in the 21st century. New York: Basic Books.

2. Shannon, A.G., 1961. "The art that is called modern." Studies. 34 (2): 67-60.

3. Hardy, G. H., 2004 [1940]. A Mathematician's Apology. Cambridge: Cambridge University Press.

4. Shannon, Anthony G., Irina Klamka, and Robert van Gend.2018. "Generalized Fibonacci Numbers and Music." Journal of Advances in Mathematics. 14 (1): 7564-7579.

5. Shannon, A.G., 1977. "Beauty and mathematics teaching." Studies. 50 (1): 45-56.

6. Bogolmolny, Alexander. 2014. https://www.cut-the-knot.org/manifesto/index.shtml.

7. Lopez, L. et al., 2008. "The Individual Video Experience (iVE): The iPod as an Educational Tool in the Museum. Art Education, January.

8. National Gallery of Art. 2019. The Elements of Art: Line, nga.gov, 2019. https://www.nga.gov/education/teachers/lessons-activities/elements-of-art/line.html.

9. Nirei, H., 2019. A Brief History of 'The Great Wave': Japan's Most Famous Artwork. Culture Trip, 2019. https://theculturetrip.com/asia/japan/articles/hokusai-s-great-wave/.

10. Beaumont-Thomas, B., 2014. "Accidental Renaissance: The Photos That Look Like Italian Paintings. "The Guardian, August 6.

\section{Authors' biographies with photos}

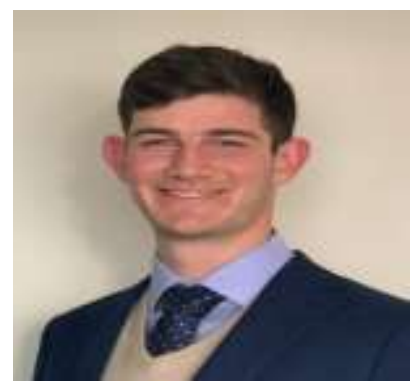

Seamus Awtel Power is a former student of mathematics at Campion College Australia, where he was taught by Professor Tony Shannon and where he graduated with a Bachelor of Arts in the Liberal Arts. He is currently working as a surgical theatre assistant and wardsman at Queensland Children's Hospital with hopes of commencing his formal studies of Medicine in 2020. This is his maiden article. He enjoys rowing, tennis, singing and political philosophy. 


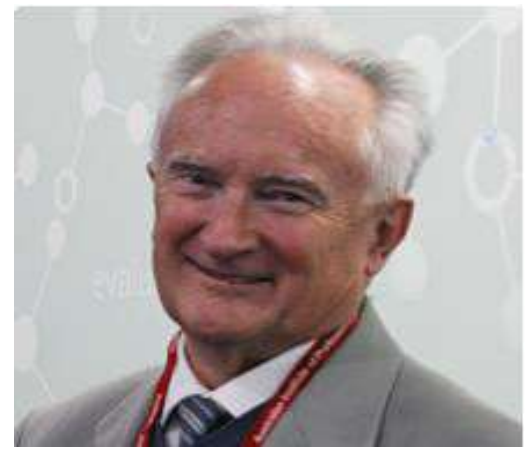

A. G. (Tony) Shannon AM is an Emeritus Professor of the University of Technology Sydney, where he was Foundation Dean of the UTS Graduate Research School and Professor of Applied Mathematics. He has also been Deputy Chancellor of the University of Notre Dame Australia, where he was awarded an honorary LLD. He also holds the doctoral degrees of PhD, EdD. and DSC. He was Master of Warrane College, the University of New South Wales, for ten years. He is co-author of numerous books and articles in medicine, mathematics, and education. He was appointed a Member of the Order of Australia (AM) in the Queen's Birthday honours list of 1987 for services to education, particularly in the field of applied mathematics. He enjoys reading, walking, theatre, watching rugby, and thoroughbred racing. 